\title{
ANNOUNCEMENT Thank you to all 2019 Mucosal Immunology Reviewers
}

\author{
Mucosal Immunology (2020) 13:383-384; https://doi.org/10.1038/s41385-020-0284-8
}

At Mucosal Immunology, we understand that a rigorous and fair peer-review process is essential to our scientific impact and reputation among scientists in our field. While our review process continues to improve, we are proud of the quality and fairness of the vast majority of reviews we receive. Below is a list of the many individuals willing to contribute to this process by providing at least one, and often many reviews during 2019. On behalf of the Mucosal Immunology editorial board, we thank you all for your time, energy, and expertise. Without you, Mucosal Immunology would not be the leading journal it is today.

Brian Kelsall Editor-in-chief

Mucosal Immunology

Abdel-Mohsen, Mohamed

Adner, Mikael

Afzali, Behdad

Ahern, Philip

Alcorn, John

Alnabhani, Ziad

Alper, Scott

Ancuta, Petronela

Arditi, Moshe

Asin, Susana

Bain, Calum

Bakaletz, Lauren

Baldwin, Troy

Baluk, Peter

Barber, Daniel

Barrett, Nora

Bartel, Sabine

Bartlett, Nathan

Basu, Joyoti

Belz, Gabrielle

Bemark, Mats

Berin, Cecilia

Beswick, Ellen

Beura, Lalit

Bianchi, Elisabetta

Bilsborough, Janine

Birchenough, George

Blumberg, Richard

Boirivant, Monica

Borish, Larry

Brandt, Eric

Brasier, Allan R.

Brazil, Jennifer

Brennan, Pattrick

Brown, Gordon

Brown, Jeremy

Byrne, Adam

Calder, Virginia

Cantorna, Margherita

Cao, Ju

Carey, Alison

Caspi, Rachel

Cauley, Linda

Cerf-Bensussan, Nadine

Cerovic, Vuk
Cerutti, Andrea

Chadee, Kris

Chai, Toby

Chamaillard, Mathias

Chen, Grace

Chen, Min

Cherrier, Marie

Chi, Hongbo

Chiang, Bor-Luen

Chiu, Chris

Choi, Jaesung

Christensen, Dennis

Clatworthy, Menna

Clavel, Thomas

Cohen, Noam

Colonna, Marco

Colucci, Francesco

Cominelli, Fabio

Cong, Yingzi

Cook, Peter

Coombes, J.

Cormier, Stephania A.

Danne, Camille

Darville, Toni

De Paiva, Cintia

De Sordi, Luisa

de Vries, Carlie Jacoba

Debes, Gudrun

Denson, Lee (Ted)

Desai, Mahesh S.

Diamond, Gill

Diefenbach, Andreas

Dillon, Stephanie

Doisne, Jean-Marc

Dong, Tao

Dorhoi, Anca

Dorin, Julia

Drummond, Rebecca

Dubyak, George

Dudeja, P.K.

Eisenbarth, Stephanie

Elson, Charles

Engel, Daniel

Erle, David

Everts, Bart

Farber, Donna

Fear, Vanessa

Feng, Carl
Foster, Paul

Fowke, Keith

Fujihashi, Kohtaro

Fulcher, Jennifer

Fulkerson, Patricia

Galletti, Jeremías

Gerlic, Motti

Geuking, Markus

Ghigo, Jean Marc

Goldstein, Daniel

Goldszmid, Romina

Gosens, Reinoud

Gotoh, Yoshiyuki

Grainger, John

Gregory, Lisa

Grivennikov, Sergey

Guery, Jean-Charles

Guo, Xiaohuan

Haldar, Pranab

Halim, Timotheus Y.F.

Hanash, Alan

Harder, Jürgen

Harker, James

Hase, Koji

Hasnain, Sumaira

Hawn, Thomas

Hayday, Adrian

Heijink, Irene

Hepworth, M.

Herbert, Debroski

Hickey, Danica

Hioe, Catarina

Hisamatsu, Tadakazu

Hoebe, Kasper

Hogan, Simon

Hölscher, Christoph

Homer, Robert

Hou, Baidong

Huckriede, Anke

Hunter, Christopher

lijima, Hideki

Ivanov, Andrei

Iwasaki, Akiko

Jacquet, Alain

Jalkanen, Sirpa

Japp, Alberto

Jay, Philippe

Jenkins, Stephen
Joeris, Thorsten

Johansson, Cecilia

Johansson, Malin

Jordana, Manel

Joyce, Sebastian

Julkunen, Ilkka

Jung, Steffen

Kaiserlian, Dominique

Kamada, Nobuhiko

Kanai, Takanori

Kao, John

Kaul, Rupert

Kedzierska, K.

Kelley, Colleen

Kelsen, Judith

Kerr, William

Khader, Shabaana

Kim, Chang

Kim, Richard

Kim, Sangwon

Kim, Yun-Gi

Kirchberger, Stefanie

Kita, Hirohito

Klatt, Nichole

Kleinjan, Alex

Klenerman, Paul

Knodler, Leigh

Knoop, Kathryn

Koch, Stefan

Kohlmeier, Jacob

Kolls, Jay

Koning, Frits

Konkel, Joanne

Koon, Hon

Krausgruber, Thomas

Krishnamoorthy, Nandini

Krueger, Andreas

Kuemmerle, Jay

Kumar, Dhiraj

Kupz, Andreas

Kurts, Chris

Kweon, Mi-Na

Lacy, Paige

Lahl, Katharina

Lash, Gendie

Lavelle, Ed

Lazarevic, Vanja

Lee, Janet 
384

Lehuen, Agnes

Leite-de-Moraes, Maria

Lelouard, Hugues

Lencer, Wayne

Lesnik, Philippe

Leto, Thomas

Lewis, George

Lewkowich, Ian

$\mathrm{Li}$, Lin-Xi

Libert, Claude

Liu, Yun-Cai

Liu, Zhanju

Liu, Zhihua

Lochner, Matthias

Longman, Randy

Lopalco, Lucia

Lorenz, Robin

Lozupone, Catherine

Lu, Wen

Luissint, Anny-Claude

Lukacs, Nicholas

Luo, Zhao-Qing

Lycke, Nils

Mabbott, Neil

MacDonald, Andrew

MacDonald, Tom

Macpherson, Andrew

Makenzie, Jason

Mani, Sridhar

Mann, Elizabeth

Mannon, Peter

Marchetti, Giulia

Martineau, Adrian

Martins, Gislâine

Masterson, Joanne

Matsuda, Akira

Matteoli, Gianluca

Maxwell, Joe

McAleer, Jeremy

McCluskey, James

McCoy, Kathy D.

McGeachy, Mandy J.

McGuckin, Michael

McKenzie, Andrew

McNicholl, Janet

McSorley, Stephen

McWilliam, Hamish

Meffre, Eric

Melgert, Barbro

Meresse, Bertrand

Michelsen, Kathrin

Milling, Simon

Mills, Kingston
Mizoguchi, Atsushi

Mjosberg, Jenny

Moffett, Ashley

Molyneaux, Phil

Monteiro, Renato

Monteleone, Giovanni

Moore, Bethany

Morrison, Richard

Mortha, Arthur

Mowat, Allan

Mucida, Daniel

Mudd, Joseph

Muir, Amanda B.

Mulvey, Matthew

Murthy, Ashlesh

Mysorekar, Indira

Nadeau, Kari

Nakajima, Hiroshi

Nakayama, Toshinori

Naren, Anjaparavanda

Nawijn, Martijn

Netea, Mihai

Neufert, Clemens

Neurath, Markus

Newberry, Rodney

Neyrolles, Olivier

Nochi, Tomonori

Nunez, Gabriel

Olive, Andrew

Ortiz, Alexandra

Paiardini, Mirko

Pandrea, Ivona

Paredes, Roger

Patel, Mickey

Peebles, Stokes

Petri, William

Petrovas, Costas

Phipps, Simon

Pizarro, Theresa

Pociask, Derek

Porcelli, Steve

Pott, Johanna

Prince, Alice

Prinz, Immo

Quiros, Miguel

Radinger, Madeleine

Rathinam, Vijay

Rauch, Isabella

Ravanetti, Lara

Re, Fabio

Reboldi, Andrea

Reeves, R. Keith

Reyes, Alejandro
Reynaud, Claude-Agnes

Rios, Daniel

Robertson, Sarah

Rodriguez-Garcia, Marta

Rodriguez, Anthony

Rothenberg, Marc

Saban, Daniel

Samsom, Janneke

Sandberg, Johan

Sandgren, Kerrie

Sauma, Daniela

Schnoor, Michael

Schughart, Klaus

Scott, Charlotte

Seré, Kristin

Shafikhani, Sasha

Sharkey, Andrew

Sheridan, Brian

Shiau, Celia

Shimamura, Michio

Shinohara, Mari

Siegers, Gabrielle

Siegmund, Britta

Siegrist, Claire-Anne

Siggers, Trevor

Sly, Laura

Smids, Carolijn

Smith, Dirk

Smith, Jason

Snapper, Scott

Snyder, Mark

Sodora, Donald

Sonnenberg, Gregory

Spencer, Jo

Spits, Hergen

St. Leger, Anthony

Stampfli, Martin

Starkey, Malcolm

Strober, Warren

Strugnell, Richard

Sukkar, Maria

Sumagin, Ronen

Sun, Jiaren

Suratt, Benjamin

Svensson Frej, Marcus

Takeda, Kiyoshi

Takei, Fumio

Tangye, Stuart

Tilburgs, Tamara

Torow, Natalia

Towne, Jennifer

Travis, Mark

Tregoning, John
Trompette, Aurélien

Trottein, Francois

Truchetet, Marie-Elise

Ubags, Niki

Uematsu, Satoshi

Ugolini, Sophie

Umetsu, Dale

Vacca, Paola

Vallance, Bruce

Van den Bossche, Jan

Van der Velden, Adrianus

Van Dyken, Steven

Van Els, Cecile

Van Kaer, Luc

Van't Land, Belinda

Varga, Steven

Veldhoen, Marc

Verreck, Frank

Vijay-Kumar, Matam

Villablanca, Eduardo

Viney, Jo

Visekruna, Alexander

Voehringer, David

Vujkovic-Cvijin, Ivan

Walsh, Patrick

Walzer, Thierry

Wan, Yisong

Watanabe, Tomohiro

Watford, Wendy

Way, Sing Sing

Wesemann, Duane

Wilen, Craig

Wilhelm, Christoph

Williams, Holly

Wilson, Mark

Winter, Nathalie

Winter, Sebastian

Woodworth, Joshua

Worthington, John

Wullaert, Andy

Wuthrich, Marcel

Xing, Zhou

$X u$, Heping

Xu, Jianying

Yan, Fang

Yang, Xuexian

Yarovinsky, Felix

Yu, Qin

Zeevi, David

Zehn, Dietmar

Zilbauer, Matthias 